\title{
PATH INTEGRAL METHOD FOR FIRST-PASSAGE PROBABILITY DETERMINATION OF NONLINEAR SYSTEMS UNDER LEVY WHITE NOISE
}

\author{
Christian Bucher ${ }^{1}$, Alberto Di Matteo ${ }^{2}$, Mario Di Paola ${ }^{2}$, and Antonina Pirrotta ${ }^{2,3}$ \\ ${ }^{1}$ Institute of Building Construction and Technology \\ Vienna University of Technology, A-1040 Wien, Karlsplatz 13/222, Austria \\ e-mail: Christian.bucher@tuwien.ac.at \\ ${ }^{2}$ Dipartimento di Ingegneria Civile, Ambientale, Aerospaziale, dei Materiali (DICAM) \\ Università degli Studi di Palermo, Viale delle Scienze, 90128 Palermo, Italy \\ \{alberto.dimatteo, mario.dipaola, antonina.pirrotta\}@e-mail.address \\ ${ }^{3}$ Department of Mathematical Sciences \\ University of Liverpool, Liverpool, UK \\ e-mail: antonina.pirrotta@liverpool.ac.uk
}

Keywords: First-passage, Path Integral method, Levy white noise, Nonlinear systems.

\begin{abstract}
In this paper the problem of the first-passage probabilities determination of nonlinear systems under alpha-stable Lévy white noises is addressed. Based on the properties of alpha-stable random variables and processes, the Path Integral method is extended to deal with nonlinear systems driven by Lévy white noises with a generic value of the stability index alpha. Furthermore, the determination of reliability functions and first-passage time probability density functions is handled step-by-step through a modification of the Path Integral technique. Comparison with pertinent Monte Carlo simulation reveals the excellent accuracy of the proposed method.
\end{abstract}




\section{INTRODUCTION}

Dynamic excitation, in many applications of engineering interest, has to be often modeled as a stochastic process, and consequently the corresponding systems response is a stochastic process too.

In this case two are the main problems commonly one has to deal with. The first one pertains the determination of the system response probability density function (PDF), the second is related to the probability the system response stays within a prescribed domain over a given time interval. The latter, generally known as survival probability or reliability function, is used to evaluate the risk of failure of the considered system and to perform the dynamical system reliability-based analysis.

Clearly, the counterpart of the above problem is the determination of the probability that the system reaches the prescribed boundary for the first time, and it is commonly named as first-passage problem [1-2].

Although first-passage problem has been extensively studied by numerous authors, even the case of a linear oscillator under white noise excitation still lacks of an exact closed form solution for its reliability function, thus demonstrating the complexity of the aforementioned issue.

Many analytical and numerical techniques have been then proposed to evaluate the reliability function or the corresponding first-passage time PDF. In this regard Monte Carlo simulation (MCS)-based approaches are among the most common numerical tools (see [3] and references therein), while other relevant contributions on the subject can be found in [4-9].

In this context, the so-called Path Integral (PI) technique, developed for the PDF determination of systems under normal and Poissonian white noise [10-13], has been modified and adapted for the solution of the first-passage problem of nonlinear systems $[5,14,15]$.

For the application of the PI technique the knowledge in closed form of the so-called conditional probability density functions (CPDFs) is required. This function, in fact, appears in the convolution integral of the Chapman-Kolmogorov (CK) equation, on which the entire PIS method is based. However, CPDFs are known in closed-form only for systems subjected to Gaussian and external Poissonian white noises [10, 12].

A third still common case of white noise exists, the so-called $\alpha$-stable Lévy white noise which belongs to the wide class of $\alpha$-stable Lévy processes.

In this regard, many real phenomena observed in diverse fields of physic and engineering show evident non-Gaussian features noticeable in heavy tails distributions, which are characteristics of the so-called $\alpha$-stable Lévy processes [16].

Due to the consequently increasing interest in the response evaluation of engineering systems under $\alpha$-stable stochastic processes, some research efforts have focused on developing numerical and analytical technique to evaluate the response of nonlinear systems with Lévy white noise input [17-20].

However, since the CPDF of nonlinear systems under a generic $\alpha$-stable Lévy white noise is not known in closed form, the PI method has not yet been applied for this kind of systems. Specifically, only the particular case of the Cauchy white noise (which belongs to the class of the Lévy white noises for $\alpha=1$ ) has been considered by Naess and Skaug [21].

In this paper the PI technique is applied and extended to cope with nonlinear systems subjected to $\alpha$-stable Lévy white noises, with any value of the stability index. Further, based on the recent application of the PI for the solution of the first-passage problem, the aforementioned technique is used to derive reliability functions and first-passage time PDFs of nonlinear systems with Lévy input. Finally, comparison with pertinent MCS is reported to assess the accuracy of the method. 


\section{REMARKS ON $\alpha$-STABLE LÉVY RANDOM VARIABLES}

In this section some definitions and properties of $\alpha$-stable random variables, which will be necessary for following derivation, are introduced.

A random variable $X$ is said to be $\alpha$-stable if, for any $\left(\rho_{1}>0, \rho_{2}>0\right)$ and independent copies $X_{1}$ and $X_{2}$ of $X$, there exist two parameters $\kappa>0$ and $\xi \in \mathbb{R}$ such that

$$
\kappa\left(\rho_{1} X_{1}+\rho_{2} X_{2}\right)+\stackrel{d}{=} X
$$

where the symbol $\stackrel{d}{=}$ indicates that the random variables $\kappa\left(\rho_{1} X_{1}+\rho_{2} X_{2}\right)+\xi$ and $X$ exhibit the same distribution [16]. The $\alpha$-stable random variable $X$ is commonly described by its Characteristic Function $(\mathrm{CF}) \phi_{X}(\theta)$, given as

$$
\phi_{X}(\theta)=E[\exp (i \theta X)]= \begin{cases}\exp \left\{i \theta \mu-\sigma^{\alpha}|\theta|^{\alpha}\left[1-i \beta \operatorname{sgn}(\theta) \tan \left(\frac{\pi \alpha}{2}\right)\right]\right\}, & \alpha \neq 1 \\ \exp \left\{i \theta \mu-\sigma|\theta|\left[1+i \beta \operatorname{sgn}(\theta) \frac{2}{\pi} \log |\theta|\right]\right\}, & \alpha=1\end{cases}
$$

where $E[\cdot]$ is the expectation operator and $\alpha \in] 0,2], \sigma>0, \beta \in[-1,1], \mu$ four real parameters, known respectively as stability index, scale, skewness and shift parameter.

It is clear then that these parameters characterize the distribution of $X$. Thus the notation $X \sim S_{\alpha}(\sigma, \beta, \mu)$ is generally used to denote the class of $\alpha$-stable random variables with the $\mathrm{CF}$ in Eq. (2). Further, for a symmetric $\alpha$-stable random variable, that is $\beta=\mu=0$, the compact notation $X \sim S \alpha S$ is adopted.

It is worth stressing that the PDFs of $\alpha$-stable random variables exist and are continuous, but unknown in explicit form with few exceptions. Specifically, a stable distribution is Gaussian when $(\alpha=2, \beta=0)$, that is $S_{2}(\sigma, 0, \mu)$, while for $\alpha<2$ only two cases are known in closed form: the Cauchy distribution when $(\alpha=1, \beta=0)$, that is $S_{1}(\sigma, 0, \mu)$; and the Lévy distribution when $(\alpha=1 / 2, \beta=1)$, that is $S_{1 / 2}(\sigma, 1, \mu)$.

Let $X \sim S_{\alpha}(\sigma, \beta, \mu)$ be an $\alpha$-stable variable and $\bar{Z}=a X+b$ a linear combination of this variable, with $a>0$ and $b$ real coefficients. It can be demonstrated that the linear combination $\bar{Z}$ is still an $\alpha$-stable random variable. That is $\bar{Z} \sim S_{\alpha}(\bar{\sigma}, \bar{\beta}, \bar{\mu})$, with

$$
\begin{array}{ll}
\bar{\sigma} & =\sigma a \\
\bar{\beta} & =\beta \\
\bar{\mu} & = \begin{cases}a \mu+b, & \alpha \neq 1 \\
(a \mu+b)-\frac{2}{\pi} \sigma \beta a \log (a), & \alpha=1\end{cases}
\end{array}
$$

It has to be noted that Eq. (3) represents a remarkable property which will be necessary for the application of the PI to the case under consideration.

As far as the $\alpha$-stable random processes are concerned, they can be defined as $\alpha$-stable random variables which depend on the parameter set $T$ [16]. 
A relevant class of $\alpha$-stable processes of engineering interest, is the one of symmetric $\alpha$ stable Lévy motion processes $\left\{L_{\alpha}(t), t \geq 0\right\}$. These are defined as zero-shift and zeroskewness Lévy motion processes, having the following properties:

i) They starts from zero with probability one, i.e.

$$
L_{\alpha}(0)=0
$$

ii) They have independent stationary increments following the $\alpha$-stable distribution

$$
L_{\alpha}(t)-L_{\alpha}(s) \sim S_{\alpha}\left((t-s)^{1 / \alpha}, 0,0\right)
$$

for any $0 \leq s<t<\infty$

iii) The CF of their increments $d L_{\alpha}(t)=L_{\alpha}(t+d t)-L_{\alpha}(t)$, takes the form

$$
\phi_{d L_{\alpha}}(\theta)=\exp \left[-d t|\theta|^{\alpha}\right]
$$

Note that, in complete analogy with the definition of the Gaussian white noise as formal derivative of the Brownian motion $B(t)$, an $\alpha$-stable Lévy white noise may be introduced as the formal derivative of a corresponding $\alpha$-stable Lévy motion, that is

$$
W_{L_{\alpha}}(t)=\frac{d L_{\alpha}(t)}{d t}
$$

Clearly for $\alpha=2$, the $\alpha$-stable Lévy white noise reverts to the Gaussian white noise. Further, the smaller the stability index $\alpha$, the greater is the departure of the Lévy white noise from the Gaussian one.

\section{PATH INTEGRAL METHOD FOR SYSTEMS UNDER $\alpha$-STABLE LÉVY WHITE NOISE INPUT}

Let a nonlinear system under an $\alpha$-stable white noise $W_{L_{\alpha}}(t)$ be given as

$$
\left\{\begin{array}{l}
\dot{X}(t)=f(X, t)+\gamma W_{L_{\alpha}}(t) \\
X(0)=X_{0}
\end{array}\right.
$$

where $f(X, t)$ is a generic nonlinear function of the response process $X(t), X_{0}$ is the initial condition, deterministic or a random with assigned PDF, and $\gamma$ is a positive constant.

The associated Ito equation associated is given as

$$
d X(t)=f(X, t) d t+\gamma d L_{\alpha}(t)
$$

in which increments $d L_{\alpha}(t)$ are characterized by the CF in Eq. (4.c).

From Eq. (8) it can be recognize that the response process $X(t)$ is Markovian. Hence the CK equation holds true, that is

$$
p_{X}(x, t+\tau)=\int_{-\infty}^{\infty} p_{X}(x, t+\tau \mid \bar{x}, t) p_{X}(\bar{x}, t) d \bar{x}
$$


where $p_{X}(x, t+\tau \mid \bar{x}, t)$ is the so-called Conditional probability density function (CPDF) and $p_{\bar{X}}(\bar{x}, t)$ is the already known PDF of $X(t)$ at a previous time instant.

Note that Eq. (8) represents the basis for the application of the PI method. However, while the CPDF for the case of normal white noise input $(\alpha=2)$ and Poissonian white noise input have already been derived in [12], for the general class of $\alpha$-stable white noise input the CPDF has not still been obtained.

To this aim, consider a new process $\bar{X}(\rho)$ ruled by the differential equation

$$
\left\{\begin{array}{l}
\dot{\bar{X}}(\rho)=f(\bar{X}, \rho)+\gamma W_{L_{\alpha}}(t+\rho) ; 0 \leq \rho \leq \tau \\
\bar{X}(0)=\bar{x}
\end{array}\right.
$$

It is evident that the only difference between Eq. (9.b) and Eq. (6.b) is that $\bar{X}(\rho)$ has a deterministic initial condition $\bar{x}$, hence the CPDF in Eq. (8) coincides with the unconditional PDF of the response process $\bar{X}(\rho)$ in Eq. (9) evaluated in $\tau$, that is

$$
p_{X}(x, t+\tau \mid \bar{x}, t)=p_{\bar{X}}(x, \tau)
$$

Supposing the time interval $\tau$ sufficiently small, it follows that an approximation of $\bar{X}(\rho)$ is given by

$$
\bar{X}(\tau)=\bar{x}+f(\bar{x}, t) \tau+\gamma d L_{\alpha}(t)
$$

In this way $\bar{X}(\tau)$ has been expressed as a linear combination of the $\alpha$-stable Lévy process $d L_{\alpha}(t)$, thus enjoying of the properties in Eqs. (3).

Specifically, since $d L_{\alpha}(t) \sim S_{\alpha}\left(\tau^{1 / \alpha}, 0,0\right)$, from Eq. (3) it immediately follows that

$$
\bar{X}(\tau) \sim S_{\alpha}\left(\gamma \tau^{1 / \alpha}, 0, \bar{x}+f(\bar{x}, t) \tau\right)
$$

Therefore, recalling Eq. (2), the CF of the process $\bar{X}(\tau)$ is given as

$$
\phi_{\bar{X}}(\theta, \tau)=\exp \left\{i \theta(\bar{x}+f(\bar{x}, t) \tau)-\gamma^{\alpha} \tau|\theta|^{\alpha}\right\}
$$

and the corresponding PDF can be evaluated by its inverse Fourier transform, that is

$$
p_{\bar{X}}(x, \tau)=\frac{1}{2 \pi} \int_{-\infty}^{\infty} \exp (-i \theta x) \phi_{\bar{X}}(\theta, \tau) d \theta
$$

It is worth stressing that, due to Eq. (10), Eq. (14) represents the CPDF for the nonlinear system under an $\alpha$-stable white noise Eq. (6).

Note that, for a generic value of the stability index $\alpha$, Eq. (14) cannot be solved in closed form, so numerical evaluation of the inverse Fourier Transform has to be performed. Only for the particular cases of $(\alpha=1)$, which corresponds to the Cauchy white noise, and $(\alpha=2)$, which corresponds to the Gaussian white noise, Eq. (14) could be solved in closed form as reported in [21]. 
Once the CPDF in Eq. (14) is obtained, the PI method may be easily implemented. Specifically, subdividing the time interval $\left[0, t_{\max }\right]$ into small intervals of equal length $\tau=\Delta t, \mathrm{CK}$ equation Eq. (8) yields

$$
\begin{aligned}
p_{X}\left(x, t_{k}+\Delta t\right) & =\int_{-\infty}^{\infty} p_{X}\left(x, t_{k}+\Delta t \mid \bar{x}, t_{k}\right) p_{X}\left(\bar{x}, t_{k}\right) d \bar{x}= \\
& =\frac{1}{2 \pi} \int_{-\infty}^{\infty} \int_{-\infty}^{\infty} \exp (-i \theta x) \exp \left\{i \theta\left(\bar{x}+f\left(\bar{x}, t_{k}\right) \Delta t\right)-\gamma^{\alpha} \Delta t|\theta|^{\alpha}\right\} p_{X}\left(\bar{x}, t_{k}\right) d \theta d \bar{x}
\end{aligned}
$$

where $t_{k}$ is a generic time instant, with $k=0, \ldots, \frac{t_{\max }}{\Delta t}$.

Starting at the time $t_{0}=0$ where $p_{X}(x, 0)$ is already known, the PDF $p_{X}\left(x, t_{1}\right)$ in the following time instant $t_{1}=0+\Delta t$ can be evaluated through Eqs. (14) and (15). This procedure can be repeated recursively until the whole time interval $\left[0, t_{\max }\right]$ is explored.

\subsection{Solution of the first-passage problem by Path Integral method}

The first-passage problem is defined as the problem of evaluating the probability that the trajectories of the response process $X(t)$ cross a prescribed boundary for the first time, within a certain interval $[0, T]$.

Once extended the PI technique to deal with systems under $\alpha$-stable white noise input, the corresponding first-passage problem can be now solved via a modification of the PIS.

Specifically, denote as $(\eta, \xi)$ the threshold barriers of the prescribed boundary, and discretize the time interval $[0, T]$ into steps of length $\Delta t$, sufficiently small so that the various trajectories are monotone in the generic interval $\left[t_{k}, t_{k}+\Delta t\right]$.

Based on the CK equation Eq. (8), let a new function, termed as reliability density function (RDF) $q_{\xi, \eta}\left(x, t_{k}+\Delta t\right)$, be introduced as

$$
q_{\xi, \eta}\left(x, t_{k}+\Delta t\right)=U(x-\eta) U(\xi-x) \int_{\eta}^{\xi} p_{X}\left(x, t_{k}+\Delta t \mid \bar{x}, t_{k}\right) q_{\xi, \eta}\left(\bar{x}, t_{k}\right) d \bar{x}
$$

where $U(\bullet)$ is the unit step function and $q_{\xi, \eta}(x, 0)=p_{X}(x, 0)$.

Note that the function $q_{\xi, \eta}\left(x, t_{k}+\Delta t\right)$ represents the probability that the trajectories of the response process $X(t)$ fall, at the generic time instant $\left(t_{k}+\Delta t\right)$, in the interval $[x, x+d x]$, and it is not a PDF since its area is not unitary.

Equation (16) represents the modification of the PI for the first-passage problem. Considering that the reliability function $R_{E}(T)$ is defined [14] as the probability that the system response $X(t)$ stays between the threshold barriers $(\eta, \xi)$ over the time interval $[0, T]$, then

$$
R_{E}(T)=\int_{\eta}^{\xi} q_{\xi, \eta}(x, T) d x
$$


Clearly the complementary to one of $R_{E}(T)$, is the probability that the system response trajectories cross the threshold barriers in the time interval $[0, T]$, and it may be termed as first-passage probability $P_{E}(T)$.

Finally, the corresponding first-passage time PDF, that is the time at which the response $X(t)$ crosses the prescribed barriers for the first time, can be obtained as

$$
p_{E}(T)=-\frac{d R_{E}(T)}{d T}
$$

Assuming that the interval $[\eta, \xi]$ is discretized such as $x_{j}=\eta+j \Delta x, j=0, \ldots, n$ and $\Delta x=(\xi-\eta) / n$, then Eq. (16) can be rewritten as

$$
q_{\xi, \eta}\left(x_{j}, t_{k}+\Delta t\right)=U\left(x_{j}-\eta\right) U\left(\xi-x_{j}\right) \sum_{r=0}^{n} p_{X}\left(x_{j}, t_{k}+\Delta t \mid \bar{x}_{r}, t_{k}\right) q_{\xi, \eta}\left(\bar{x}_{r}, t_{k}\right) \Delta x
$$

where it has been assumed that the discretization over the domain $x$ in $\left(t_{k}+\Delta t\right)$ is the same as that of $\bar{x}$ at time $t_{k}$.

It can be noted that Eq. (18) can be rewritten in compact form as

$$
\boldsymbol{q}\left(t_{k}+\Delta t\right)=\boldsymbol{T}\left(t_{k}+\Delta t, t_{k}\right) \boldsymbol{q}\left(t_{k}\right)
$$

in which $\boldsymbol{q}\left(t_{k}+\Delta t\right)$ and $\boldsymbol{q}\left(t_{k}\right)$ are vectors whose elements are given by $q_{\xi, \eta}\left(x_{j}, t_{k}+\Delta t\right)$ and $q_{\xi, \eta}\left(\bar{x}_{r}, t_{k}\right)$ respectively, while $\boldsymbol{T}\left(t_{k}+\Delta t, t_{k}\right)$ is a matrix whose elements $T_{j, r}$ are

$$
T_{j, r}=U\left(x_{j}-\eta\right) U\left(\xi-x_{j}\right) p_{X}\left(x_{j}, t_{k}+\Delta t \mid \bar{x}_{r}, t_{k}\right) \Delta x
$$

If the excitation process is stationary, the matrix $\boldsymbol{T}\left(t_{k}+\Delta t, t_{k}\right)$ depends on $\Delta t$ only and it can be computed once before hand. Thus the entire PIS is just a sequence of matrix-vector multiplications, and Eq. (20) yields

$$
\boldsymbol{q}\left(t_{k}+\Delta t\right)=\boldsymbol{T}(\Delta t) \boldsymbol{q}\left(t_{k}\right)
$$

\section{NUMERICAL EXAMPLE}

In this section the solution for the first-passage problem of a nonlinear oscillator under an $\alpha$-stable Lévy white noise $W_{L_{\alpha}}(t)$ is reported. Specifically, let the nonlinear system be given as

$$
\left\{\begin{array}{l}
\dot{X}(t)=-a X(t)-\varepsilon|X(t)|^{\rho} \operatorname{sgn}[X(t)]+W_{L_{\alpha}}(t) \\
X(0)=0
\end{array}\right.
$$

The nonlinear system considered represents a wide class of systems, depending on the values of the parameters $(a, \varepsilon, \rho)$ considered. In this paper the chosen parameter of the system are: $(a=1.2, \varepsilon=1.5, \rho=0.8)$. Further, as far as the $\alpha$-stable Lévy white noise is concerned, 
the chosen value of the stability index is $(\alpha=1.7)$. Note that the oscillator in Eq. (23) is quiescent for $t \leq 0$, thus the PDF in $t=0$ is a Dirac's delta.

Numerical solutions obtained through the proposed method have been obtained considering a step size $(\Delta t=0.01 \mathrm{~s})$, while the accuracy of the PI technique has been assessed through comparison with pertinent MCS with 60000 samples. Note that MCS has been carried out as reported in [22].

In Fig. (1) the RDF of the system in Eq. (23) is depicted for a value of the upper bound $(\xi=1)$ and different values of the time $T$.

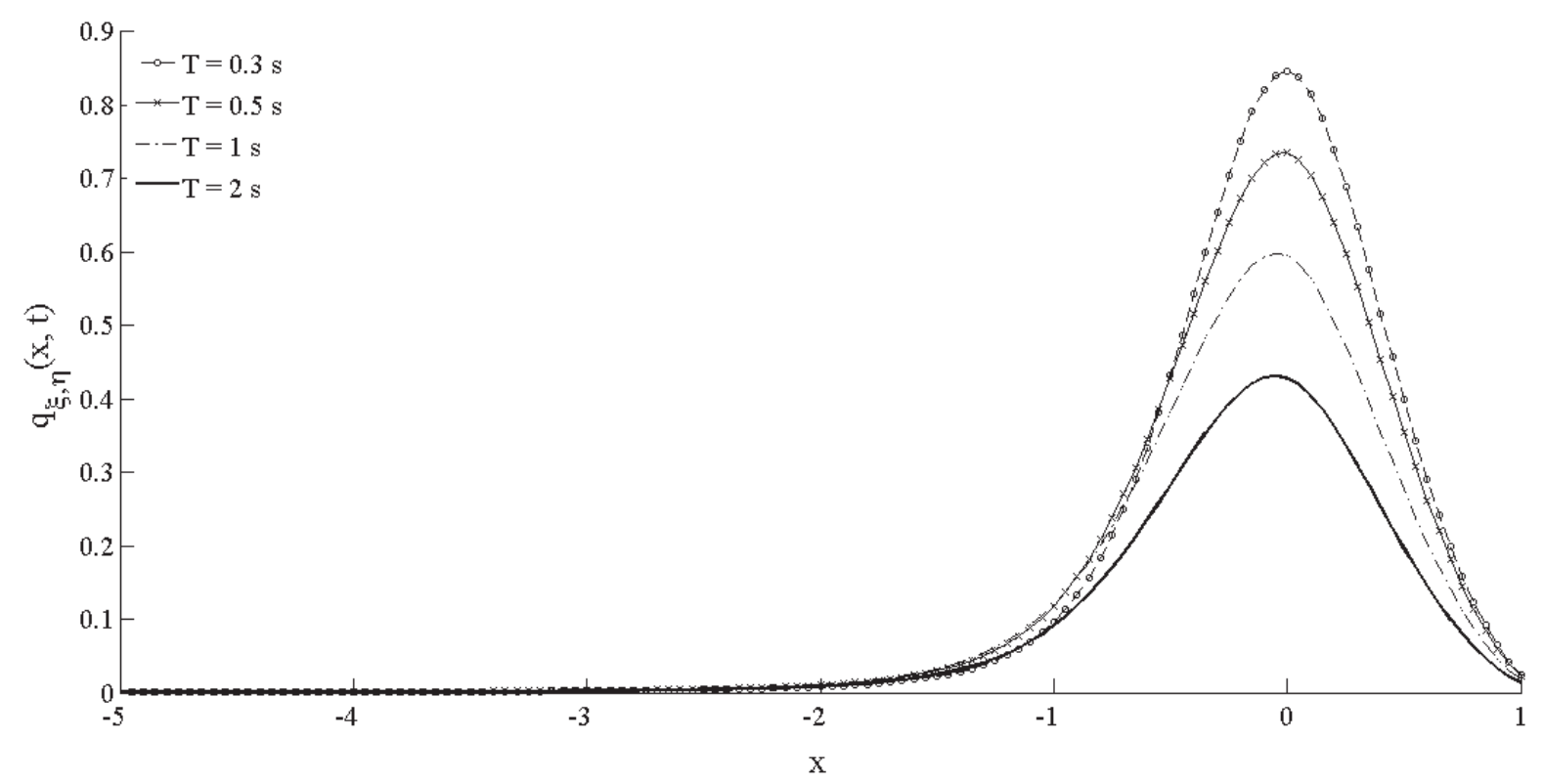

Figure 1: Reliability density function of the system in Eq. (23).

In order to show the accuracy of the proposed method for several values of the upper threshold $\xi$, in Fig. (2) the first-passage probabilities $P_{E}(T)$ are shown for a total time $(T=2 s)$ and different values of $\xi$. 


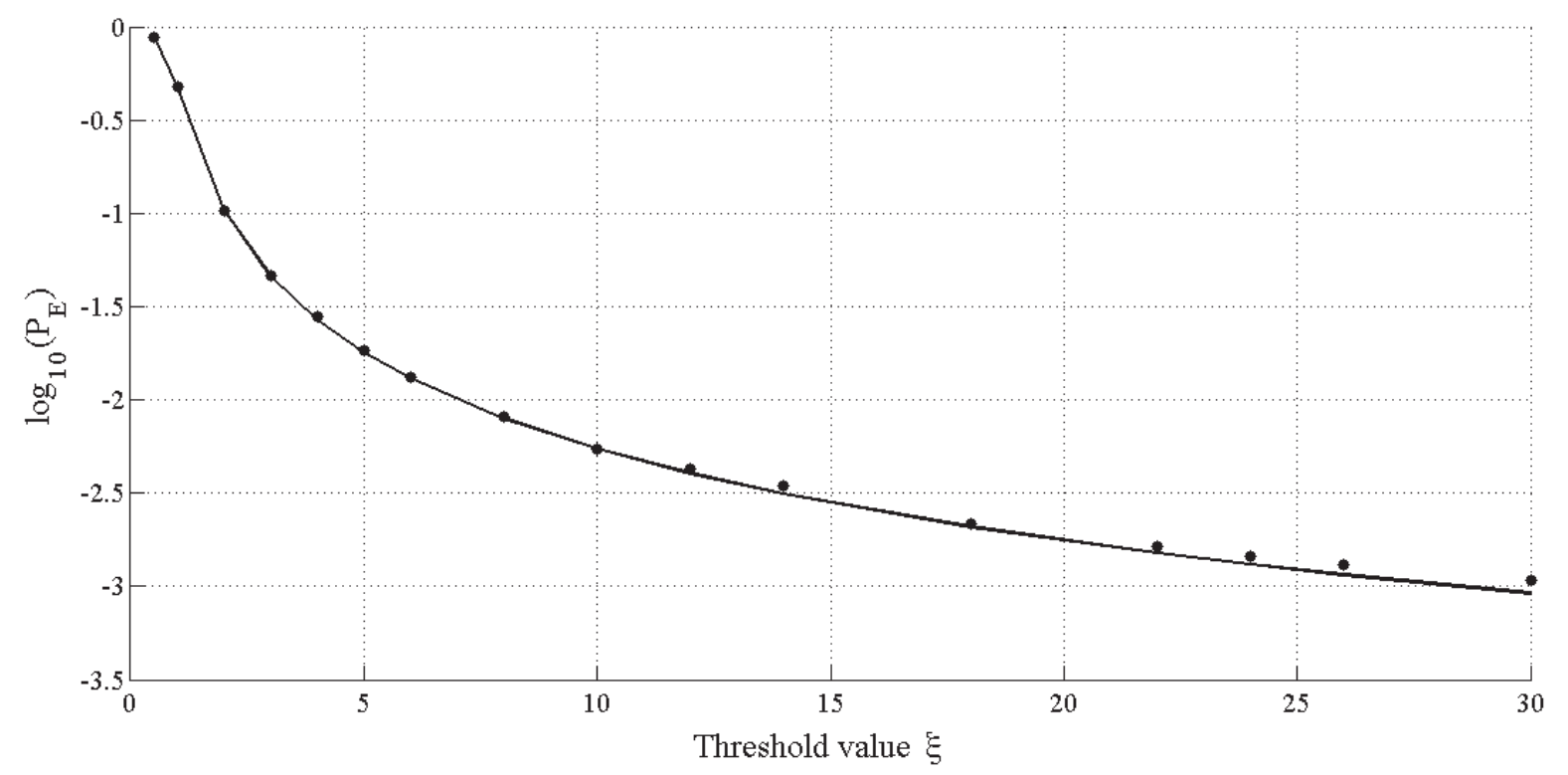

Figure 2: First-passage probabilities for system in Eq. (23). Continuous line - Proposed method; Black dots MCS.

As it can be seen, the PI technique provides very accurate results even for very large values of the threshold.

Finally, PI based first-passage time PDFs $p_{E}(T)$ vis-à-vis MCS data are plotted in Fig. (3) for three different values of the upper threshold $\xi$.

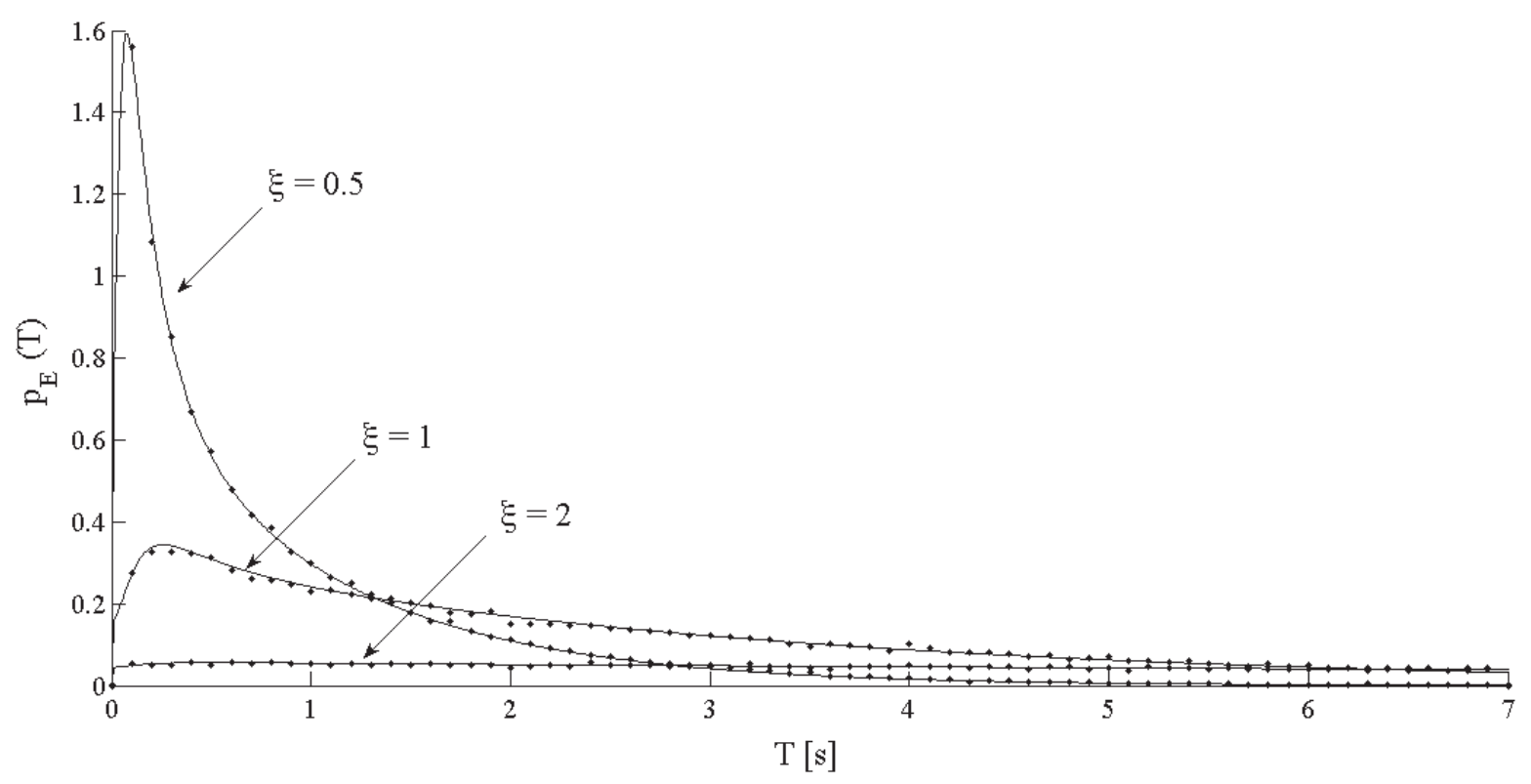

Figure 3: First-passage time PDF for system in Eq. (23). Continuous line - Proposed method; Black dots - MCS.

The excellent agreement between PI solution and MCS results, shown in Fig. (3), assess the accuracy of the proposed procedure at any value of the total time $T$. 


\section{CONCLUSIONS}

- The Path Integral technique has been extended to deal with nonlinear systems under $\alpha$ stable Lévy white noise.

- The appropriate Conditional PDF for systems driven by Lévy white noise is introduced, for any value of the stability index $\alpha$.

- Specifically the Conditional PDF is found as inverse Fourier Transform of the corresponding Characteristic Function, which is known in closed form.

- The Path Integral procedure has been modified in order to obtain the reliability density functions and consequently the Reliability function of the nonlinear systems under $\alpha$ stable Lévy white noise.

- Comparison whit pertinent Monte Carlo Simulation has shown that the proposed procedure leads to really accurate results for any value of the barrier level and total time considered, even for large value of the nonlinearity.

\section{REFERENCES}

[1] Y.K. Lin, G.Q. Cai, Probabilistic Structural Dynamics. McGraw-Hill, New York, USA, 1995.

[2] J.B. Roberts, P.D. Spanos, Random vibration and statistical linearization. Wiley, New York, USA, 1990.

[3] G.I. Schueller, H.J. Pradlwarter, P.S. Koutsourelakis, A critical appraisal of reliability estimation procedures for high dimensions, Probabilistic Engineering Mechanics, 19, 463-474, 2004.

[4] P.D. Spanos, Survival probability of non linear oscillators subjected to broad band random disturbances, International Journal of Non-Linear Mechanics, 17, 303-317, 1982.

[5] I.A. Kougioumtzoglou, P.D. Spanos, Response and First-Passage Statistics of Nonlinear Oscillators via a Numerical Path Integral Approach, Journal of Engineering Mechanics, 139, 1207-1217, 2013.

[6] H.U. Köylüoglu, S.R.K. Nielsen, R. Iwankiewicz, Reliability of non-linear oscillators subjected to Poisson driven impulses, Journal of Sound and Vibration, 176, 19-33, 1994.

[7] L. Bergman, B. Spencer, First passage time for linear systems with stochastic coefficients, Probabilistic Engineering Mechanics, 2, 46-53, 1987.

[8] C. Bucher, Asymptotic sampling for high-dimensional reliability analysis, Probabilistic Engineering Mechanics, 24, 505-510, 2009.

[9] C. Bucher, M.T. Sichani, S. Nielsen, Efficient estimation of first passage probability of high-dimensional nonlinear systems, Probabilistic Engineering Mechanics, 26, 539-549, 2011.

[10] A. Naess, V. Moe, Efficient path integration method for nonlinear dynamic systems, Probabilistic Engineering Mechanics, 15, 221-231, 2000. 
[11] D.V. Iourtchenko, E. Mo, A. Naess, Response probability density functions of strongly non-linear systems by the path integration method, International Journal of Non-Linear Mechanics, 41, 693-705, 2006.

[12] M. Di Paola, R. Santoro, Path integral solution for non-linear system enforced by Poison White Noise, Probabilistic Engineering Mechanics, 23, 164-169, 2008.

[13] A. Pirrotta, R. Santoro, Probabilistic response of nonlinear systems under combined normal and Poisson white noise via path integral method, Probabilistic Engineering Mechanics, 26, 26-32, 2011.

[14] D.V. Iourtchenko, E. Mo, A. Naess, Reliability of strongly nonlinear single degree of freedom dynamic systems by the path integration method, Journal of Applied Mechanics, 75, DOI: 10.115/1.2967896, 2008.

[15] I.A. Kougioumtzoglou, P.D. Spanos, Stochastic response analysis of softening Duffing oscillator and ship capsizing probability determination via numerical path integral approach, Probabilistic Engineering Mechanics, 35, 67-74, 2014.

[16] G. Samorodnitsky, S.M. Taqqu, Stable non-gaussian random processes. Stochastic models with infinite variance. Chapman and HAll/CRC, USA, 1994.

[17] M. Grigoriu, Linear systems subjected to non-Gaussian $\alpha$-stable processes, Probabilistic Engineering Mechanics, 10, 23-34, 1995.

[18] M. Di Paola, G. Failla, Stochastic response of linear and non-linear systems to $\alpha$-stable Lévy white noises, Probabilistic Engineering Mechanics, 20, 128-135, 2005.

[19] M. Di Paola, A. Pirrotta, M. Zingales, Ito calculus extended to systems driven by $\alpha$ stable Lévy white noises (a novel clip on the tails of Lévy motion), Probabilistic Engineering Mechanics, 42, 1046-1054, 2007.

[20] M. Grigoriu, Equivalent linearization for systems driven by Lévy white noise, Probabilistic Engineering Mechanics, 15, 185-190, 2000.

[21] A. Naess, C. Skaug, Extension of the numerical path integration method to filtered $\alpha$ stable Levy noise, Computational Stochastic Mechanics, Santorini, Greece, 1999.

[22] A. Pirrotta, Non-linear systems under parametric white noise input: Digital simulation and response, International Journal of Non-Linear Mechanics, 40, 1088-1101, 2005. 